\title{
Covid-19. Diseño antropométrico de silla y escritorio para recibir clases de posgrado en línea.
}

\author{
Covid-19. Anthropometric design of chair and desk to receive online \\ graduate classes.
}

\author{
Manuel Armando Alencastro Chuez. ${ }^{1}$, Manolo Alexander Córdova Suarez. ${ }^{2}$, Esthela \\ del Rocío Freire Ramos. ${ }^{3} \&$ María Fernanda Cuenca Cajamarca. ${ }^{4}$
}

\section{DOI: https://doi.org/10.33262/anatomiadigital.v4i3.1.1885}

\begin{abstract}
.
Introduction. The Covid-19 pandemic toppled the global economy and common ways of working, forcing students and teachers to take classes online in conditions that are not ergonomically suitable for their health. Objective. Anthropometrically design a chair and desk for a group of students receiving online graduate classes and mitigate ergonomic risk. Methods. It began by determining the real dimensions of the
\end{abstract}

\section{Resumen.}

Introducción. La pandemia por Covid19 doblegó a la economía mundial y las maneras comunes de trabajar, obligando a los estudiantes y maestros a recibir clases en línea en condiciones que no son adecuadas ergonómicamente para su salud. Objetivo. Diseñar antropométricamente una silla y un escritorio para un grupo de estudiantes que reciben clases de posgrado en línea y atenuar el riesgo ergonómico. Métodos. Se

\footnotetext{
${ }^{1}$ Universidad Regional Autónoma de los Andes, Posgradista. Ambato-Ecuador. Guayaquil-Ecuador. pg.manuelaac52@uniandes.edu.ec, D ORCID: https://orcid.org/0000-0001-5048-243X

2 Universidad Nacional de Chimborazo, Facultad de Ingeniería, Riobamba-Ecuador, manolo.cordova@unach.edu.ec ORCID: https://orcid.org/0000-0001-6786-7926

${ }^{3}$ Universidad Regional Autónoma de los Andes, Posgradista. Ambato-Ecuador. Médico General. Hospital General Puyo. Puyo-Ecuador. esthe_01ef@hotmail.com D ORCID: https://orcid.org/0000-0003-08913747

${ }^{4}$ Universidad Regional Autónoma de los Andes, Posgradista. Ambato-Ecuador. mafer1600@hotmail.com (D) ORCID: https://orcid.org/0000-0002-7353-0227
} 
main anthropometric elements that determine a group of professionals who study online and then applying percentile calculations to choose the best dimension of 14 parts of a chair and a desk that mitigates the level of ergonomic risk. Results. From the application of the calculation of the percentiles for a population of 35 students considering a class mark (m) of 6 for 14 anthropometric dimensions that coincide with 14 important parts of the design, the following dimensions were chosen: seat height $42.49 \mathrm{~cm}$, space between legs and table $17.39 \mathrm{~cm}$, seat depth $44.68 \mathrm{~cm}$, table height $53.93 \mathrm{~cm}$, armrest height $22.81 \mathrm{~cm}$, upper back width $43.49 \mathrm{~cm}$, table top depth $60,17 \mathrm{~cm}$, bottom table depth 70.43 $\mathrm{cm}$, distance allowing mobility $93.64 \mathrm{~cm}$, width of seat base $38.91 \mathrm{~cm}$, distance between armrests $45.88 \mathrm{~cm}$, height of seat back $60.93 \mathrm{~cm}$. Conclusion. An ergonomic workspace was designed considering real anthropometric dimensions for a group of graduate students studying in teleworking conditions due to the Covid-19 pandemic.

Keywords: Anthropometric design, interior spaces, ergonomics, online classes. empezó determinando las dimensiones reales de los elementos antropométricos principales que determinan un grupo de profesionales que estudian en línea para luego aplicando cálculo de percentiles escoger la mejor dimensión de 14 partes de una silla y un escritorio que atenúe el nivel de riesgo ergonómico. Resultados. De la aplicación del cálculo de los percentiles para una población de 35 estudiantes considerando una marca de clase (m) de 6 para 14 dimensiones antropométricas que coinciden con 14 partes importantes del diseño se escogió las siguientes dimensiones: altura del asiento $42,49 \mathrm{~cm}$, espacio entre las piernas y mesa 17,39 $\mathrm{cm}$, profundidad del asiento $44,68 \mathrm{~cm}$, altura de la mesa $53,93 \mathrm{~cm}$, altura reposabrazos $22,81 \mathrm{~cm}$, ancho superior del espaldar 43,49 $\mathrm{cm}$, profundidad superior de la mesa $60,17 \mathrm{~cm}$, profundidad inferior de la mesa $70,43 \mathrm{~cm}$, distancia que permite movilidad 93,64 $\mathrm{cm}$, ancho de la base del asiento $38,91 \mathrm{~cm}$, distancia entre reposabrazos $45,88 \mathrm{~cm}$, altura del espaldar del asiento 60,93 cm. Conclusión. Se diseñó un espacio de trabajo ergonómico considerando dimensiones antropométricas reales para un grupo de estudiantes de posgrado que estudia en condiciones de teletrabajo por pandemia de Covid-19.

Palabras clave: Diseño antropométrico, espacios interiores, ergonomía, clases en linea.

\section{Introducción.}

La pandemia por Covid-19 causó cambios estructurales en las maneras de trabajar de las personas en todo el mundo (Orejuela, 2020). Este nuevo panorama dio ocasión a la promoción y desarrollo de modalidades de estudio que estaban en crecimiento o que simplemente no se las consideraba como prioridad como son las clases en línea 
(Santillan, 2020). Pero estos espacios de trabajo improvisados incrementaron las molestias osteomioarticulares y enfermedades psicosociales en todos los ocupantes de estos nuevos puestos de trabajo (García-Salirrosas \& Sánchez-Poma, 2020). El teletrabajo y las modalidades de enseñanza solo intentaron compensar la justificación de su uso con el incremento de indicadores de cumplimiento y productos verificables en tiempo real que complicaron la situación del factor humano (Chavez Escobedo, Martínez Macías, \& Dávila Garza, 2020). En las universidades las clases se tornaron virtuales y nuevas modalidades de educación fueron adoptadas sin una planificación que incluya mobiliario y periféricos que den soporte y prevengan enfermedades ocupacionales (Calvo, Cervi, Tusa, \& Parola, 2020). Los estudiantes y profesores ocuparon el mobiliario disponible en sus casas, sillas de cuatro soportes y mesas como escritorios de trabajo (Bengtsson et al., 2020).

Las lesiones por ocupar espacios reducidos o fuera de los alcances biomecánicos aceleran los procesos peligrosos que causan enfermedades osteomioarticulares (Chaverra Rojas, 2019). Estos desordenes músculo esquelético son fuente principal de las dolencias que presentan los trabajadores que incluyen enfermedades de los músculos, tendones, vainas tendinosas, síndromes de atrapamientos nerviosos, alteraciones articulares y neurovasculares (García-González, Chiriboga-Larrea, \& Vega-Falcón, 2021). El libro de diagnóstico y tratamiento de las alteraciones del movimiento define estas molestias como dolorosas por daño miofascial, periarticular o de los tejidos articulares originados por microtraumatismos y sobre esfuerzos (Robaina Aguirre, León Palenzuela, \& Sevilla Martínez, 2000).

El diseño ergonómico de los espacios interiores ayuda a establecer los planos de trabajo y los elementos que se adaptan a las condiciones de un grupo que ocupa ese entorno (Flores, 2001). Esta técnica que es parte de la ergonomía estudia las proporciones del cuerpo humano, peso, volumen, centro de gravedad, para lograr la adaptación física entre el cuerpo humano y el entorno que lo rodea (Mondelo, Bombardo, Busquets, \& Torada, 2004). Conocer las medidas de ciertos segmentos del cuerpo de los trabajadores y relacionarlos con las partes del mobiliario y equipos que ocupará el trabajador garantizan un diseño pensado en el ocupante no solo para evitar daños que pueden originar las malas posturas de trabajo, movimientos repetitivos, sobreesfuerzos, sino también accidentes laborales (Párraga Velásquez, 2014).

La antropometría del diseño utiliza la estadística para recomendar valores que caen dentro de grupos o clases preestablecidas que se replican y son representativas de estas muestras (Párraga Velásquez, 2014). Estas dimensiones calculadas se denominan percentiles o medidas de posición no central que ayudan a dividir la distribución de los datos de una población en número de segmentos o cuantiles (Claros Collazos \& Ramírez González, 2017). Este concepto proporciona la ubicación de orden de un sujeto en el conjunto de todos los datos.

Este trabajo tiene como objetivo diseñar una silla y un escritorio para uso de un grupo de estudiantes en modalidad en línea, considerando los elementos más relevantes que se 
relacionan a las partes principales de estos espacios interiores y que garanticen confort ergonómico al mayor número de personas que lo ocuparán (Trujillo Huber, Pereira Despaigne, Jacas García, \& García Díaz, 2016).

\section{Metodología}

\section{Diseño antropométrico}

La antropometría usa las dimensiones las personas a una base de comparaciones. Su aplicación al diseño se centra en la adaptación de la interfase del cuerpo humano y los componentes del equipo o espacio interior necesita datos antropométricos de los ocupantes para escoger el percentil de mejor adaptación. La finalidad del equipo o espacio a destinar debe considerar las normas de seguridad y prevención preestablecidas en las regulaciones locales. Sin embargo, cuando se disponen de los datos de una población el diseño solo se centra en determinar el percentil adecuado de cada segmento del espacio a diseñar. En esta investigación no se tomaron datos antropométricos de otras poblaciones o de poblaciones similares para no tener errores de diseño, sino que se partió de las mediciones reales de los individuos que ocuparán la silla y el escritorio.

En la primera etapa se utilizó un plan de medición de los datos estructurales y funcionales del cuerpo humano y la selección de las medidas requeridas. Se empezó con: a) determinación de las facilidades considerando que el espacio donde se efectuarán la toma de mediciones debe ser privado y debe estar adecuado con todos los implementos necesarios, es recomendable utilizar cuartos separados cuando más de un sujeto está siendo medido, b) el equipo de medición debe contar con los instrumentos antropométricos necesarios: antropómetro, varillas curvas para antropómetro, calibrador extensible, compás deslizable, cinta antropométrica y además el equipo de cómputo necesario y los formatos para anotaciones, c) cada medición que vaya a efectuarse a un individuo requiere del trabajo de dos analistas: uno para efectuar físicamente la medición y otro para realizar las anotaciones, es recomendable también que los hombres midan a los hombres y las mujeres a su mismo sexo también, e) las personas encargadas de la medición, necesitan un entrenador o capacitador, para que les valide el hecho de estar utilizando la misma técnica de medición y anotación.

En la segunda etapa se ordenó los datos en una recopilación inicial y una selección de repetición para determinar la frecuencia. Luego se determinó la extensión de las variables continuas y se clasificó o agrupó por intervalos de clases. El número de intervalos o subgrupos se calcularon siguiendo la ecuación de Sturger. Ver ecuación:

$$
m=1-3.3 *(\log . n)
$$

Ecu: 1

Donde:

$\mathrm{m}=$ número de intervalos o clases

$\mathrm{n}=$ número de personas 
Una vez determinado la amplitud de las clases, se debe determinar cuál es el extremo inferior y cuál el superior de cada una de las clases. Ese interrogante lo resuelve calculando la amplitud de clase (C) con la ecuación 2.

$$
C=\frac{L s-L i}{m}
$$

Donde:

$\mathrm{C}=$ amplitud

Ls= límite superior

$\mathrm{Li}=$ límite inferior

$\mathrm{m}=$ clases

Luego se procede a calcular los percentiles de los arreglos realizados para ello se busca la clase donde se encuentra el valor de $\mathrm{k}$ en las tablas de frecuencias acumuladas así:

$$
P_{k}=\frac{\frac{k . N}{100}-F_{i-1}}{f_{i}} \cdot a_{i}
$$

Donde:

$\mathrm{P}_{\mathrm{k}}=$ Percentil

$\mathrm{F}_{\mathrm{i}-1}=$ frecuencia acumulada anterior a la clase del percentil

$\mathrm{a}_{\mathrm{i}}=$ amplitud de clase

\section{Consideraciones de diseño}

Para la elección de los percentiles se tomó en cuenta:

\section{Diseño de la silla.}

Se considerará un percentil de la altura de la parte superior de la superficie de asiento respecto al suelo no muy grande para evitar compresión y perturbación de la circulación sanguínea en la cara inferior de los muslos. Además, el percentil de la holgura entre la planta del pie y el suelo mínimo para mejorar la estabilidad del cuerpo. Se tomará el percentil de altura del asiento no muy pequeño para evitar que las piernas pueden extenderse y echarse hacia delante y los pies quedan privados de toda estabilidad. Para el percentil de la altura poplítea estará en relación con 5, para incluir al sector de población con dimensiones más pequeñas de cuerpo. Para la profundidad del asiento se consideró un percentil pequeño para que el borde o arista frontal del asiento no comprima la zona posterior de las rodillas y no entorpezca el riego sanguíneo a piernas y pies.

La longitud nalga-poplíteo es la que nos dará la profundidad de asiento idónea. Escogemos una largura nalga-poplíteo de 5 percentil, $44.68 \mathrm{~cm}$. Por consiguiente, cualquier profundidad que exceda de $39.63 \mathrm{~cm}$, no acomodará a los usuarios más bajos, mientras que esta, proporcionaría una silla confortable con el $95 \%$ de los mismos. Para dotar de apoyo a la región lumbar de la silla se tomó en cuenta la prominencia de la zona 
de las nalgas. Aunque el tamaño, es una de las consideraciones más relevantes, el perfecto acoplamiento usuario-silla es el componente más arduo de determinar.

Para suministrar soporte a la región lumbar de tamaño pequeño se buscó el perfil espinal, singularmente en la zona lumbar. Se evitó que el acoplamiento sea tan completo que impida cambiar la posición del cuerpo. Basta con proporcionar un apoyo congruente a la zona lumbar a toda la espalda por lo que se escogió el percentil 5 en las dimensiones distancia de hombros, nalga poplítea, ancho de cadera y así asegurar el respaldo adecuado tanto a personas pequeñas como a las más altas de nuestra muestra, mientras que para la altura del asiento se tomó el percentil 50 de la altura de mitad de hombro sentado para asegurar en esta ocasión a las personas altas. Para nuestro diseño la silla estará correctamente regulada cuando los brazos se apoyen sobre la mesa o plano de trabajo de una forma estable. Se tomó un ángulo de flexión de 90 a 110 , para mantener los hombros bajos y relajados. Para el reposapiés, los pies no se apoyarán en el suelo. Se escogió un ángulo de 110 grados de inclinación para nuestra silla.

\section{Diseño de mesa}

Se aseguró una altura de la mesa a la altura de los codos cuando está sentado y que los codos queden en descanso y flexionados $90^{\circ}$. Además, para dejar un espacio libre suficiente bajo la mesa para acomodar al usuario y que la superficie del tablero tenga el tamaño suficiente para colocar todos los elementos necesarios para llevar a cabo la tarea de manera cómoda se escogió el percentil 50 para la profundidad de la mesa, mientras que para la altura escogemos el percentil 99.

\section{Resultados}

\section{Resultados del diseño de la silla y escritorio}

En la tabla 1. se observa la selección de los percentiles calculados y escogidos para cada segmento de la silla y el escritorio diseñado.

Tabla $\mathbf{N}^{\circ}$ 1. Resultado del diseño de la silla y escritorio

\begin{tabular}{llccccc}
\hline \multicolumn{1}{c}{$\begin{array}{c}\text { Dimensión } \\
\text { antropométrica }\end{array}$} & \multicolumn{1}{c}{ Parte de diseño } & P1 & P5 & P50 & P95 & P99 \\
\hline Altura Poplítea & Altura del asiento & 40,02 & 42,49 & 46,74 & 50,41 & 50,87 \\
Altura Muslo & $\begin{array}{l}\text { Espacio entre las piernas y la } \\
\text { mesa }\end{array}$ & 10,46 & 11,65 & 14,92 & 17,39 & 17,85 \\
Nalga Poplítea & Profundidad del asiento & 39,63 & 44,68 & 47,40 & 49,68 & 49,91 \\
Rodilla & Altura de la mesa & 42,81 & 46,07 & 52,19 & 53,93 & 55,56 \\
Codo Reposo & Altura reposabrazos & 20,11 & 20,57 & 22,81 & 26,24 & 29,41 \\
Hombros & Ancho superior del espaldar & 40,69 & 43,49 & 49,35 & 51,72 & 51,94 \\
Nalga Rodilla & $\begin{array}{l}\text { Profundidad superior de la } \\
\text { mesa }\end{array}$ & 47,15 & 47,81 & 52,09 & 60,17 & 60,83 \\
Nalga punta de Pie & $\begin{array}{l}\text { Profundidad inferior de la } \\
\text { mesa }\end{array}$ & & & & & \\
Nalga Pierna & $\begin{array}{l}\text { Distancia que permite } \\
\text { movilidad }\end{array}$ & 80,57 & 62,91 & 70,43 & 73,19 & 78,82 \\
& & 86,25 & 87,30 & 93,64 & 100,54 & 102,94
\end{tabular}


Tabla $\mathbf{N}^{\circ}$ 1. Resultado del diseño de la silla y escritorio (continuación)

\begin{tabular}{llccccc}
\hline $\begin{array}{l}\text { Dimensión } \\
\text { antropométrica }\end{array}$ & Parte de diseño & P1 & P5 & P50 & P95 & P99 \\
\hline $\begin{array}{l}\text { Ancho de Caderas } \\
\text { Anchura Codo a }\end{array}$ & Ancho de la base del asiento & 38,17 & 38,91 & 46,76 & 58,71 & 59,73 \\
$\begin{array}{l}\text { Codo } \\
\text { Altura Mitad }\end{array}$ & $\begin{array}{l}\text { Alturancia entre reposabrazos del espaldar del } \\
\text { asiento }\end{array}$ & 45,28 & 45,88 & 47,19 & 49,26 & 49,84 \\
Hombro Sentado & 55,07 & 55,41 & 60,93 & 64,75 & 64,94 \\
Altura Ojo Sentado & $\begin{array}{l}\text { Distancia que permite } \\
\text { movilidad }\end{array}$ & 60,18 & 60,96 & 73,95 & 79,46 & 79,88 \\
Posición Sedente & $\begin{array}{l}\text { Distancia que permite } \\
\text { movilidad }\end{array}$ & 80,37 & 81,89 & 89,20 & 92,45 & 92,88 \\
\hline
\end{tabular}

Nota: Los percentiles se calcularon considerando tres mediciones de cada segmento escogido. Las letras representan cada parte del cuerpo para facilidad de la toma de datos.

En la figura 1. Se observa los datos del diseño en diferentes posiciones y de todas las vistas.

Figura 1. Detalle de silla y escritorio antropométrico

Bípedo vista axial Bípedo vista sagital




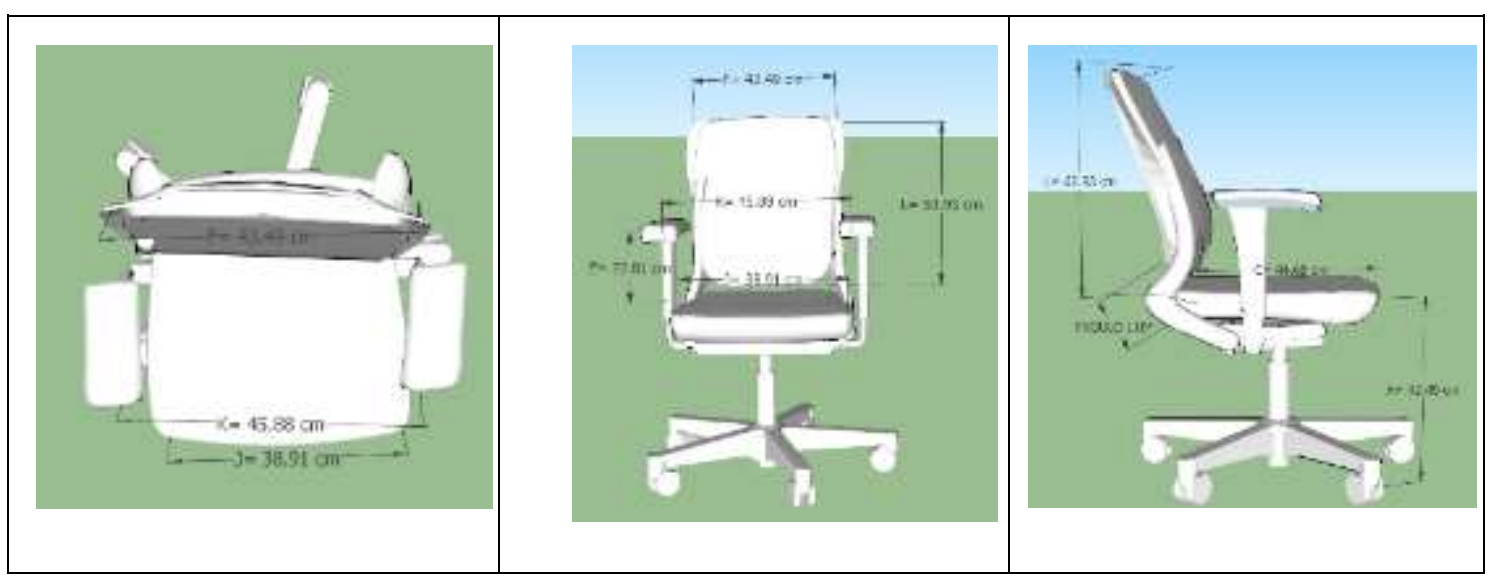

Nota: Los resultados son calculados. No son percentiles tomados de otros estudios.

En la figura 2. Se observa los datos del diseño de la silla y escritorio en vista axial, sagital y coronal.

Figura 2. Detalle de silla y escritorio antropométrico

\begin{tabular}{|l|l|l|l|}
\hline MESA VISTA AXIAL & MESA VISTA SAGITAL & $\begin{array}{l}\text { SILLA } \\
\text { CORONAL }\end{array}$ & VISTA \\
\hline & & & \\
\hline
\end{tabular}

Nota: Los resultados son calculados. No son percentiles tomados de otros estudios.

\section{Discusión}

Para tener un resultado más exacto se debería utilizar datos y mediciones distinguiendo género, ya que a pesar de que en nuestro país la variación de las magnitudes mesurables antropométricas no es mucha.

Se debería tomar percentiles de poblaciones estudiadas, pero en Ecuador no existen, lo que limita al diseñador a utilizar de poblaciones cercanas. La limitación de este cálculo es que la población es cambiante lo que implica actualizar los datos y renovar los diseños.

\section{Conclusiones}

- Se determinó que lo correcto es trabajar en base a percentiles, sustituyendo la elección de las medidas a medias aritméticas ya que no puede existir grupos homogéneos y las variantes pueden complicar el uso de mobiliario prediseñado.

- Se diseñó un espacio de trabajo ergonómico considerando dimensiones antropométricas reales para un grupo de estudiantes de posgrado que estudia en 
condiciones de teletrabajo por pandemia de Covid-19.

- Se analizó la teoría sobre antropometría de diseño de espacios interiores, y se dedujo que es importante que el análisis se lo haga de manera específica, en cada puesto de trabajo, puesto que no es lo mismo para una silla gerencial como para una recepción, ya que cada unidad de trabajo posee sus propias necesidades.

- Se definió la importancia del análisis y diseño de un puesto de trabajo, para salvaguardad la integridad física tanto como la emocional del trabajador, quien en muchas de las ocasiones está expuesto a largas jornadas laborales.

\section{Referencias bibliográficas}

Bengtsson, A. M., Bugallo, L., Coccoz, V., D'Adamo, P., Lozada, M., Méndez, L. M., . . . Salsa, A. M. (2020). "La casa convertida en mundo" como contexto de aprendizaje en tiempos de pandemia.

Calvo, S. T., Cervi, L., Tusa, F., \& Parola, A. J. R. L. d. C. S. (2020). Educación en tiempos de pandemia: reflexiones de alumnos y profesores sobre la enseñanza virtual universitaria en España, Italia y Ecuador. (78), 1-21.

Chaverra Rojas, M. E. (2019). Diseño de puestos de trabajo seguros y saludables. Corporación Universitaria Minuto de Dios,

Chavez Escobedo, J. M., Martínez Macías, J. G., \& Dávila Garza, R. J. R. D. (2020). Educación a Distancia y Teletrabajo. 15(1).

Claros Collazos, A. S., \& Ramírez González, A. D. (2017). Diseño de un prototipo de robot bípedo para la configuración antropométrica de sus percentiles.

Flores, C. (2001). Ergonomía para el diseño: Designio Teorpia y práctica.

García-González, C. A., Chiriboga-Larrea, G. A., \& Vega-Falcón, V. J. R. I. C. (2021). Prevalencia de enfermedad osteomioarticular lumbosacras y miembros inferiores en auxiliares de enfermería. 100(3).

García-Salirrosas, E. E., \& Sánchez-Poma, R. A. (2020). Prevalencia de trastornos musculoesqueléticos en docentes universitarios que realizan teletrabajo en tiempos de COVID-19. Paper presented at the Anales de la Facultad de Medicina.

Mondelo, P. R., Bombardo, P. B., Busquets, J. B., \& Torada, E. G. (2004). Ergonomía 3: Diseño de puestos de trabajo (Vol. 3): Universitat Politecnica de Catalunya. Iniciativa Digital Politecnica.

Orejuela, J. J. P. (2020). Cuarto espíritu del capitalismo: pandemia y malestar.

Párraga Velásquez, M. d. R. E. (2014). Diseño ergonómico de aulas universitarias que permitan optimizar el confort y reducir la fatiga de estudiantes y docentes. 
Robaina Aguirre, C., León Palenzuela, I. M., \& Sevilla Martínez, D. J. R. C. d. M. G. I. (2000). Epidemiología de los trastornos osteomioarticulares en el ambiente laboral. 16(6), 531-539.

Santillan, W. J. C. R. d. d. c. d. 1. U. T. I. (2020). El teletrabajo en el COVID-19. 9(2), 65-76.

Trujillo Huber, J. C., Pereira Despaigne, O. L., Jacas García, C., \& García Díaz, R. d. 1. C. J. M. (2016). Silla de escritorio ergonómica. 20(10), 2258-2266.

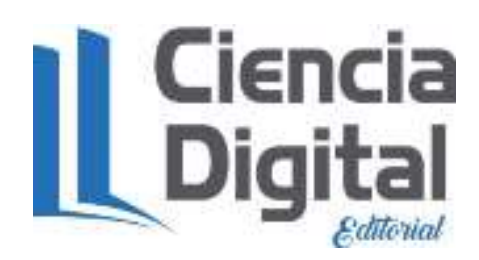




\section{PARA CITAR EL ARTÍCULO INDEXADO.}

Alencastro Chuez, M. A., Córdova Suarez, M. A., Freire Ramos, E. del R., \& Cuenca Cajamarca, M. F. (2021). Covid-19. Diseño antropométrico de silla y escritorio para recibir clases de posgrado en línea. Anatomía Digital, 4(3.1), 107-117. https://doi.org/10.33262/anatomiadigital.v4i3.1.1885

\section{\Ciencia}

El artículo que se publica es de exclusiva responsabilidad de los autores y no necesariamente reflejan el pensamiento de la Revista Anatomía Digital.

El artículo queda en propiedad de la revista y, por tanto, su publicación parcial y/o total en otro medio tiene que ser autorizado por el director de la Revista Anatomía Digital.
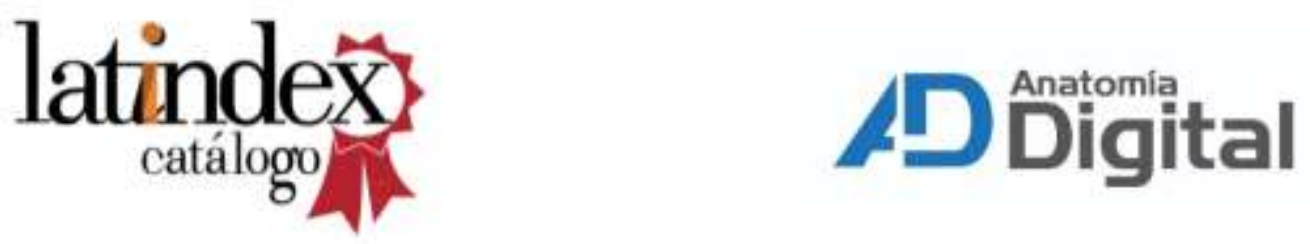